\title{
Histopathology and the future
}

\author{
D. L. GARDNER \\ From the Division of Experimental Pathology, Kennedy Institute, London
}

SYNOPSIS A plea is made for a revolution in diagnostic histopathology. In the present account a brief analysis is given of the drawbacks to existing methods used in the histopathological laboratory and suggestions are made for research in those methods which could lead to progress in the speed and sensitivity of diagnosis and to great economies in histopathological practice. The abolition or replacement of certain techniques used at present is proposed.

With the exception of a page or two of tables, less than $0.2 \%$ of the recent Association of Clinical Pathologists' 70-page memorandum on 'Data processing in laboratory services' (1968) was devoted to a consideration of histopathology, and the possible automation of histopathology received equally scant consideration at a recent Symposium on Automation and Data Processing in Pathology (College of Pathologists, 1969). Yet a substantial proportion of the diagnostic effort of Health Service and of university pathology laboratories is devoted to this subject which, in many centres, is by tradition controlled by the consultant in administrative charge. The advent of Skegg's auto-analyser has radically changed the face of clinical chemistry (Nelson, 1969) and is influencing the emerging pattern of diagnostic haematology and of microbiology. By contrast, histopathology, upon which much informed surgical opinion relies for diagnostic precision, is in the doldrums and some doubt whether it will survive the next decade as an individual discipline.

The time is therefore ripe for a reappraisal of both the technical and professional aspects of

${ }^{1}$ Based on a talk given at an Association of Clinical Pathologists Symposium on the 'Emerging pattern of pathology services' held at St Stephen's Hospital, London, on 17 January 1969.

Received for publication 1 May 1969. histopathology in laboratory diagnosis, and in the present account I propose to examine current histopathological practice, comment on its problems, and make proposals for changes which could, within the next five to seven years, restore histopathology to its former place as a leader of medical laboratory diagnostic technique.

\section{Evolution of Histopathology and its Decline}

The great advances in pathological knowledge made during the second half of the 19th century rested upon the proper use of tissue sectioning and on the study of material microscopically but these advances have been overshadowed by the growth of immunology, of microbiology, and of biochemistry. A virtual standstill in technical development in pathological histology now contrasts with an explosive growth of technical methods in these sister subjects.

Histopathology rests upon the four techniques of optical microscopy, introduced in the 1820 s with the advent of the achromatic compound microscope; on paraffin embedding, widely used since its adoption in the latter part of the last century; on section cutting, made possible by the perfection of microtomes in the middle of the 19th century; and on staining, a process evolving 
from studies of dyes synthesized in Germany during the 1870 s. For 100 or more years, therefore, hospital pathologists of most countries have been using diagnostic methods which have remained substantially unchanged and no relevant technical advance has been made since 1900 .

The histopathologist must continue to advise on the extent and spread of a malignant tumour, on the presence or absence of inflammation in an appendix, and on the cyclical changes present in the endometrium; he is almost powerless to aid the clinician when a disease process causes illness on a molecular or submolecular scale, a situation revealed when, for example, death quickly follows coronary artery obstruction or insufficiency. In these circumstances no recognizable change may be found in heart muscle, the contractile function of which has abruptly ceased. Yet it remains a a simple matter for the biochemist to demonstrate that the enzyme pathways of such a portion of heart muscle are no longer normal and that oxidative phosphorylation and ionic balance are grossly impaired.

It is no exaggeration to say therefore that histopathology is at a crossroads, but at a different junction from that described by Professor Cappell in 1960. Unless the histopathologist can devise methods which rapidly and efficiently shed light on numerous widespread sensitive but critical changes in cell and tissue function, which at the moment lie beyond the grasp of his technology, the subject as a whole will surely but inevitably disintegrate, to be replaced or engulfed by the disciplines of biochemistry, immunology, and biophysics.

It is therefore necessary to analyse the reasons why histopathology is floundering and why physicians, who in the 1920s and 1930s thronged the postmortem room to sit at the feet of the great morbid anatomists of that time, now prefer to read the typewritten reports emerging from the computer-linked autoanalyzers of the biochemists.

\section{What are the Present Techniques of Histopathology and Why are They in Decline?}

When a portion of tissue is despatched by the surgeon to the pathological laboratory, the arrival of the specimen is followed more or less quickly by a process of documentation and description which can never be wholly replaced. Thereafter the tissue may be rapidly frozen for study in cryostat section or, in most instances, is plunged into a protein denaturant, called by convention a 'fixative', in order to prepare it for the timehonoured process of embedding in paraffin wax and subsequent coloration by an organic dye. Fixation is slow, messy, cumbersome, spaceconsuming, and destructive; it preserves the tissue at the cost of rendering impossible any of the other modern forms of investigation such as histochemistry, enzyme-activity determinatio@s, and immunofluorescence; the perpetuation of paraffin embedding overlooks the growth of the science of plastics; 'staining' is little changed fre the days when the Florentine merchants of the sixteenth century dyed their imported cloth. $\overrightarrow{\vec{\omega}}$

The fixed tissue is slowly but surely dehydrated and impregnated with a paraffin we्s; at present, the process of dehydration is of eien completed overnight in a 'histokine' whamch permits technicians' time to be saved and fीe tissue to be ready the morning after receipt. Unless the tissue specimen is extremely small the process is likely to take upwards of 10 to 12 hours. Impregnation with wax is followed by the mant operation of embedding which may be accelerated by using a simple vacuum device. The embed block of tissue, properly orientated, is now refdy for the anachronistic technique of cutting,,$-a$ procedure dating from the hand-held razors $\overrightarrow{\&}$ f the early 19th century and involving the use of a microtome. Tissue cutting is time-consuming, demands considerable expertise, and is impossibje to automate: technicians skilled in its practice 앵 becoming increasingly scarce.

The cut sections, placed on the surface ofa glass slide, are now freed from paraffin wax dy a process of clearing and the section, once again in water, is ready for staining. In general terriss, the organic dyes used for staining differ lit from those which fascinated Ehrlich and whinh evolved from the dyes synthesized by his unce, Weigert. The purpose of the dyes is to give different components of the sections individual cologrs to allow easy optical distinction between nuclear and cytoplasmic components. Staining is, by ofl counts, an art rather than a science, controled with difficulty and involving considerable ti⿳⺈:日e and much training.

The stained tissue is now covered with $\underline{3}_{\mathbf{a}}$ mountant such as the older Canada balsign which has many desirable properties or the optically less desirable synthetic media which dyy quickly. The glass slide with its stained section is now labelled and numbered for despatch to expectant pathologist.

A large proportion of the work of the hisfopathology laboratory of today uses these of fashioned, costly methods as a basis for urgent diagnostic technique of extreme practical importance. Much of the laboratory resouress are used during only 38 hours each week. Grantefif, the cryostat is coming into its own, allowifing rapidly frozen tissue to be quickly cut and stained; certainly, cytodiagnosis can sometimes be placed on an automated basis; and some smill portions of tissue can be embedded and staingd by hand to yield material for rapid diagnosis. But there remains the overriding impression of a methodology ripe for evolution and change, poised for radical technical development and, In the absence of this development, due for inevitagie elimination. 
What Can be Done to Change the Present Technology of Histopathology?

I have seen few papers describing research into methods of fixation in recent years, with the exception of those devoted to the study of sections prepared for the electron microscope. Surely it should be possible to use the new knowledge of organic chemistry to find substances which will fix rapialy and expeditiously. Could not a pressurized vessel be employed, exposing the thin block of tissue to a formalin or glutaraldehyde vapour at great pressure for short times? A programme of research along these lines should allow the development of a rapid technique for fixation so that tissues are ready for dehydrating and embedding within no more than five to 10 minutes of receipt. Alternatively, cannot fixation be eliminated? This has already been done in the case of cryostat material, but cannot the new knowledge of the plastic industries be used to find a material which impregnates as it fixes?

Given a plastic of this kind, the way should be paved for the elimination of that symbol of mid-19th century pathology, the microtome. Ultrasonic and laser beam techniques are now widely used in cutting metals, plastics, and ceramics for industrial use, and the combination of a suitable plastic with an ultrasonic pencil should permit the automated cutting of thin sections for histopathology. There is already no need to mount tissue sections on glass slides; long ribbons of pliable plastic have been evolved for this purpose and allow chains of sections to be packed in coils which can be unwound like cinefilm. Plastic-embedded material could be handled in the same way.

The use of stains in histopathology rests on the assumption that the light microscope is to be used for diagnostic study. Little or no research has been undertaken to determine whether the standard light microscope can be replaced by the phase-contrast or polarizing microscope allowing unstained material to be used for diagnosis. If this proved possible in a reasonable proportion of instances, staining procedures could be eliminated or at least greatly reduced in frequency.

It will be seen that a main obstacle to radical change in histopathological diagnosis is the supposed need for the light microscope. What can be done to change this view?

Very little attention has been given until recently to the possibility of studying material microscopically in three-dimensional representation rather than in two-dimensional. The growing use of the scanning electron microscope is now showing that, at magnifications as low as $\times 30$, the whole of a substantial sized specimen can be viewed entirely in focus, in three dimensions. Thus an object as large as the head of a guinea-pig femur can be viewed completely, all parts being in focus and the surface studied in detail at increasing magnification. It should prove possible to place much histopathological diagnosis on a rapid survey of material by scanning electron microscopy. The material to be used for this purpose could be rapidly frozen and dehydrated before being coated with a thin film of metal; alternatively, it could be examined after rapid surface fixation in osmic acid followed by coating. In either case, and notwithstanding the time necessary for fixation or freezing with subsequent drying, there would appear to be at least a possibility of eliminating a proportion of the current histopathological technique and replacing it by three-dimensional studies.

The proposed method of stereo-histopathology could only be developed if it were run in parallel with current techniques for a trial period of, say, five years in the first instance. Scanning electron microscopes will become smaller and cheaper but this new approach would demand the rewriting of current texts in pathology. The morbid anatomist teaches his student with gross specimens mounted to show three-dimensional aspects of diseased tissues but, until very recently, has made no attempt to display three-dimensional structure on a microscopic scale (Elias and Pauley, 1966).

The attraction of phase-contrast microscopy for the study of tissue sections is partly the elimination of the need for staining but partly the readiness with which black and white images can be adapted to automatic scanning microscopy. Some advances have been made in the adaptation of automatic scanning light microscopes for cytological diagnosis but this method is still under trial. If the tissue section for histopathological use could be viewed as a black and white image, the prospects of a satisfactory automatic microscopic technique appear to be appreciably improved. The ideal case to consider during a period of research and development in this field of technology is the undecalcified bone section stained by the von Kossa silver technique. Consideration shows that the section to be viewed by an automatic scanning microscope would comprise areas which are either black, unstained, or coloured, the choice of colour being arbitrary. A pencil of light could scan the entire section or the section would be moved at stated intervals and speeds in front of the pencil of light by a type of motorized stage already commercially available. Light of selected wavelengths can be measured, amplified and recorded, and the amounts of light at these wavelengths used to give measures of the relative proportions of calcified and uncalcified bone matrix.

The application of automated techniques of this kind to the diagnosis of stained sections of tissues such as, for example, the appendix or stomach, offers very much greater difficulties; it does not seem possible at the present time to foresee any rapid advance in this approach. If it proved possible for, say, the 20 salient features characterizing an inflamed appendix to be 
recorded on computer tape by one observer in each of 1,000 examples of appendicitis, then when the 1001st appendix section was scanned by automatic microscope, the unknown case could be related to the content of the memory store in terms of the 20 features recorded. It must be clear at once that this approach would take many years to establish and involve much laborious preparation in order to build up memory stores relating to the common disease processes likely to be encountered by the histopathologist. I can see no other approach to this question.
Proposals for Future Histopathology Laboratory Design

In the accompanying diagram an attempt is made to suggest a design for a histopathology. laboratory in the group or teaching hospita表 laboratory of the 1980 s.

Specimens received quickly from the surgeo would be described by the professional pathole ogist in the manner used at the present time. The descriptions, recorded verbally, would be in corporated into a memory store and computeo

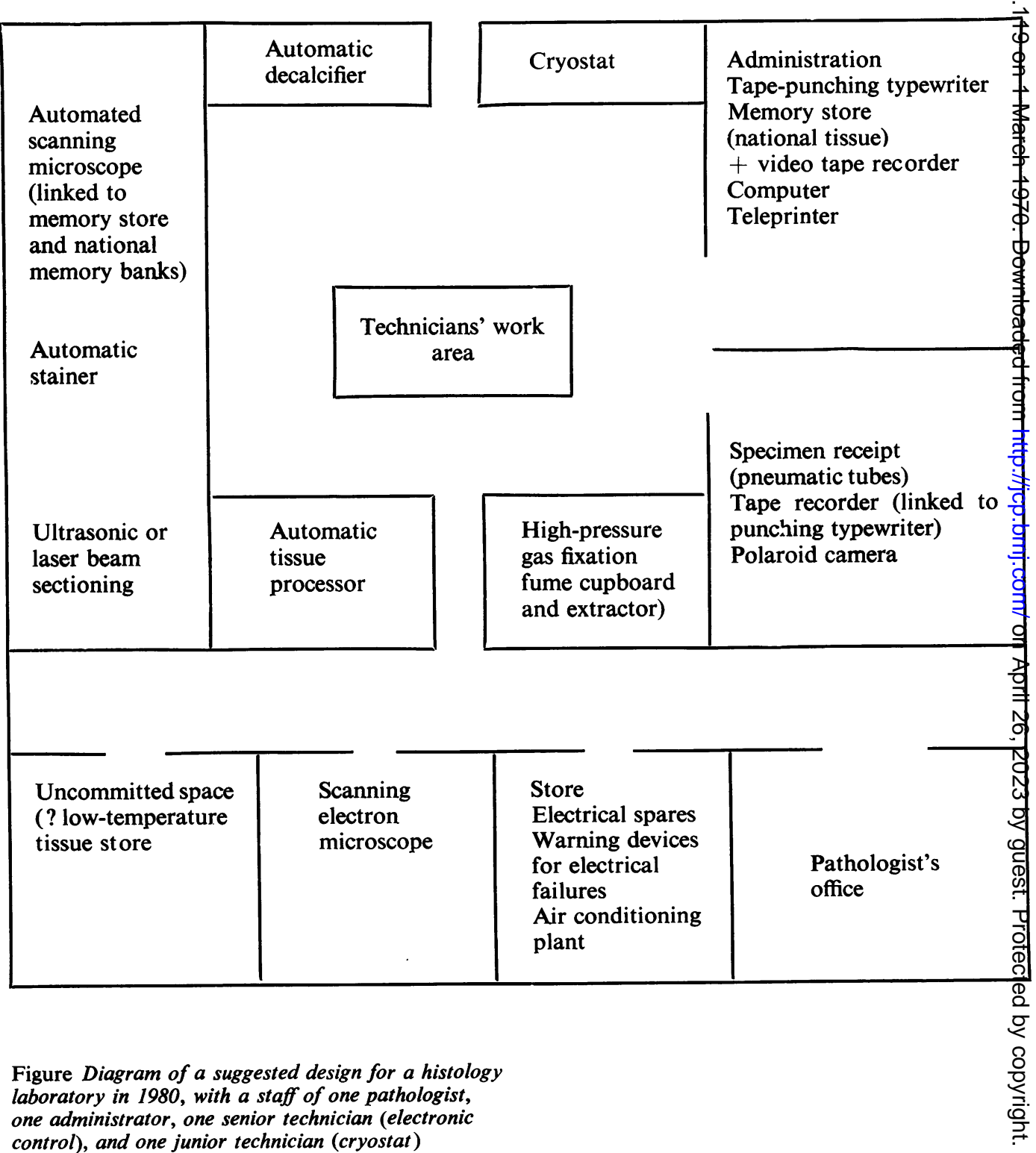


tape would be punched by typewriter linked to the tape. Video tape would be used to record the appearance of the material and the visual image would be available for recall together with the verbal. Alternatively, polaroid film could be used routinely to prepare photographic records.

The specimens, trimmed to a convenient size, could be passed quickly to a technician for rapid freezing before cryostat sectioning or, in most instances, to a rapid high-pressure fixation tank. Five to 10 minutes later the fixed tissue would be placed in an automatic processor of a type already available on the market and within 60 to 90 minutes would be ready for cutting. On the assumption that paraffin is still in use, a knife would continue to be employed to prepare tissue sections. If, however, the whole process of embedding could be revolutionized by the adoption of a quick-setting plastic, then the use of ultrasonic or laser cutting is envisaged.

The unstained section would be viewed by conventional illumination or under phase-contrast or polarizing conditions with an automated microscope. Where stained material from common disease processes was scanned, the findings would be related to those contained in a regional or national memory store to which the laboratory would be linked. Material requiring special treatment, as, for example, for the elimination of calcium, would be automatically decalcified before embedding.

The teaching hospital laboratory would use a compact scanning electron microscope to record three-dimensional images and would relate these to the new knowledge of three-dimensional pathology which must evolve in the coming 10 years. A microprobe analyser incorporated in this instrument would permit the measurement of any chosen element at points selected microscopically. Reserves of tissue would no longer consist of bulky tanks of evil-smelling formalin but would be helium or liquid nitrogen cold stores or low-temperature storage cabinets. The laboratory would require substantial stores of electrical spares and the technician in charge would undoubtedly receive training in electronics as part of his normal course.

Subsidiary procedures using qualitative and quantitative histochemical methods will evolve in the same way as those described above and it appears likely that a proportion of histological diagnoses will be made on this basis. There appears no particular reason, for example, why the diagnosis of myocardial infarction or appendicitis should not be made by homogenizing representative samples of tissue or by conducting conventional analyses of selected enzyme activities in single freeze-dried sections of tissue; these sections can be prepared quickly and within three hours of the death of a patient from coronary artery disease, the physician should have in his hand the figures for the activities of three or four selected myocardial enzymes. Immunofluorescent techniques would be used to complement the methods already described, their practicability resting very largely on the speed with which they can be conducted on cryostat snap-frozen material.

\section{References}

Association of Clinical Pathologists (1968). Data Processing in Laboratory Services.

Cappell, D. F. (1960). Pathologists at the crossroads. Lancet, 2 863-867.

College of Pathologists (1969). Symposium on Automation and Data Processing in Pathology. J. clin. Path., 22, suppl. (Coll. Path.) 3.

Elias, H., and Pauley, J. E. (1966). Human Microanatomy. Davis, Philadelphia.

Nelson, M. G. (1969). Automation in the laboratory. J. clin. Path., 22, 1-10. 